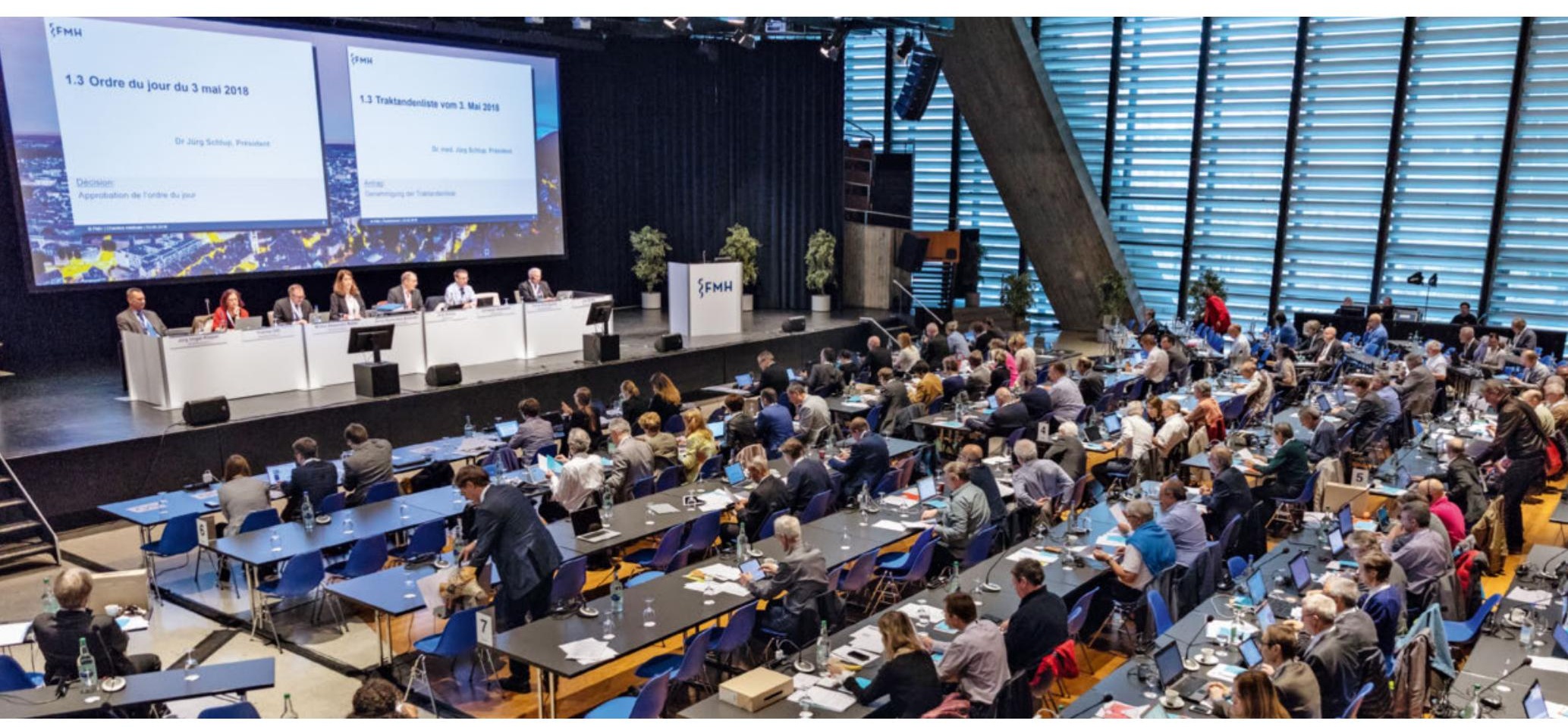

Die Ärztekammer-Delegierten als Vertreter ihrer Basis- und Fachorganisationen.

\title{
Beschlussprotokoll der ersten Ärztekammer 2018
}

\section{Monika Henzen}

Leiterin Abteilung Zentrales Sekretariat

\section{Mittwoch, 2. Mai 2018}

\section{Begrüssung, Mitteilungen, Bestellung des Büros}

Jürg Schlup/Präsident FMH begrüsst alle Delegierten und die eingeladenen Gäste zur ersten Ärztekammer 2018.

Nach den üblichen organisatorischen Mitteilungen durch Anne-Geneviève Bütikofer/Generalsekretärin FMH wird das Büro bestellt. Dieses besteht aus dem Präsidenten, dem Vizepräsidenten, der Generalsekretärin, Julien Duruz/RD FMH als Leiter Stimm- und Wahlbüro, Anne-Sylvie Thiébaud/RD FMH als stellvertretende Leiterin Stimm- und Wahlbüro, und den nachfolgenden mit 105 Ja, 2 Nein und ohne Enthaltungen gewählten Stimmenzählenden:

Alexander Zimmer, Markus Bremgartner, Jean-Pierre Grillet, Florian Leupold, Hans-Anton Vogel, Regula Capaul, Lucia Zürcher, Pierre Arnold, Carlos Munoz, Anja Zyska Cherix

\section{Genehmigung Traktandenliste}

Antrag:

Genehmigung der Traktandenliste.

Beschluss:

Die Traktandenliste wird einstimmig genehmigt.

Zu Beginn jeder Sitzung legt die ÄK gemäss Art. 11, Abs. 3 GO die Zeit fest, nach deren Ablauf weder Beschlüsse gefasst noch Wahlen vollzogen werden dürfen. Der Präsident schlägt als Tagungsende 18.00 Uhr vor.

Beschluss:

Der Antrag auf Festlegung des Tagungsendes auf 18.00 Uhr wird einstimmig angenommen.

Weiter stellt der Vorsitzende den Ordnungsantrag, die Anträge für die heutige ÄK schriftlich einzureichen. Art. 11, Abs. 5 der GO sieht vor, dass jeder Delegierte, jedes ZV-Mitglied und der Generalsekretär das Recht 


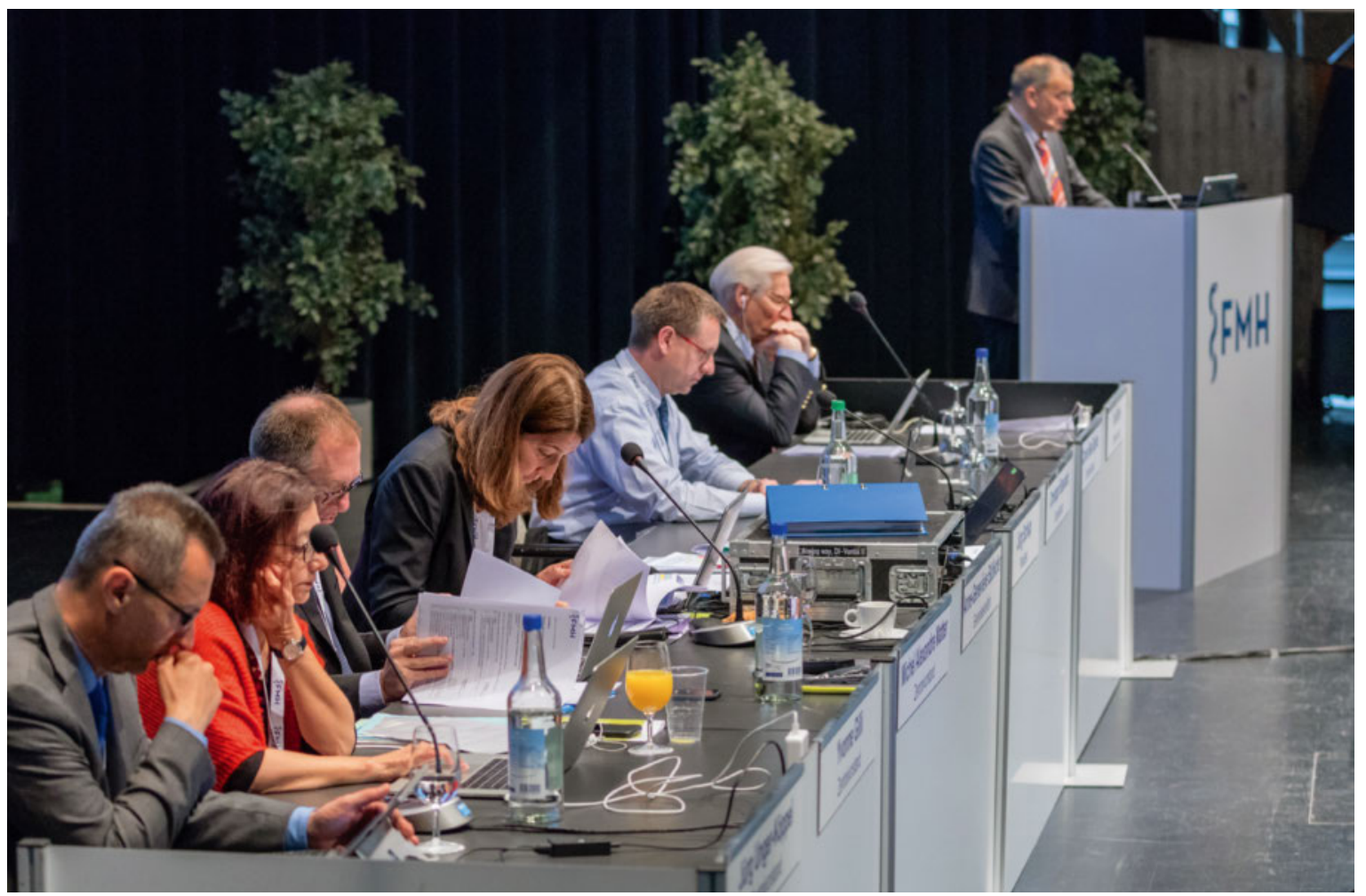

Der FMH-Präsident Jürg Schlup begrüsst die Anwesenden zur ersten Ärztekammer 2018. Zu seiner Rechten der Zentralvorstand und die Generalsekretärin.

haben, Anträge zu den zur Diskussion stehenden Traktanden zu stellen. Anträge sind dem Vorsitzenden schriftlich vor oder während der Sitzung oder ausnahmsweise mündlich einzureichen.

Ordnungsantrag: Schriftliche Einreichung der Anträge

Abänderungsvorschläge und Anträge sind dem Vorsitzenden schriftlich einzureichen. Mündliche Anträge werden nicht entgegengenommen und sind ungültig.

Beschluss:

Der Antrag wird mit 105 Ja, 3 Nein und 10 Enthaltungen gutgeheissen.

\section{Tarife}

\subsection{Projekt TARCO - Verabschiedung Leistungsstruktur (Nomenklatur) und weiteres Vorgehen}

Jürg Schlup/Präsident FMH erläutert, dass es bei den heute zur Abstimmung vorgelegten Anträgen um die ärzteinterne konsolidierte Tarifstruktur TARCO geht, zu welcher sich die rund 90 angeschlossenen Ärzteorganisationen äussern konnten. Die Delegierten entscheiden an der ÄK im Oktober 2018 über die mit den Tarifpartnern bereinigte ambulante Tarifstruktur inklusive Taxpunkte.
Urs Stoffel/ZV-Mitglied und Departementsverantwortlicher Ambulante Versorgung und Tarife informiert über die Ausgangslage und den aktuellen Stand des Projektes. Nach der Ablehnung der Urabstimmung vom 9.6.2016 hat die DV am 26.1.2017 das zuständige Departement mit dem Projekt TARCO beauftragt. Mit TARCO werden die Ziele nach einer konsolidierten, gesetzeskonformen, betriebswirtschaftlichen und sachgerechten Tarifstruktur (gemäss Art. 43, Abs. 4 KVG) wie auch die Sicherung der Tarifautonomie verfolgt. Er erinnert daran, dass nach dem Scheitern der gemeinsamen Revision von 2016 der Bundesrat am 18.8.2017 per 1.1.2018 zum zweiten Mal in die Tarifautonomie eingegriffen hat. Dieser Eingriff, der aus Sicht der FMH weder sachgerecht noch betriebswirtschaftlich ist, hat einen grossen Einfluss auf die heute zur Diskussion stehende Leistungsstruktur. Zum ersten Tarifeingriff im 2014 hat das Bundesgericht zwischenzeitlich einen Entscheid gefällt. Dass sich der Bundesrat nach Ansicht des Bundesgerichts von politischen Anliegen leiten lassen darf, während sich die Tarifpartner strikt an die Vorgaben des KVG halten müssen, hat die Ärzteschaft mit Befremden zur Kenntnis genommen. Umso wichtiger ist es nun für die FMH, die Ziele der Gesamtrevision des TARMED weiterzuverfolgen und zusammen mit den Tarifpartnern eine revidierte, sachgerechte und betriebswirtschaftliche Tarifstruktur bis Ende 


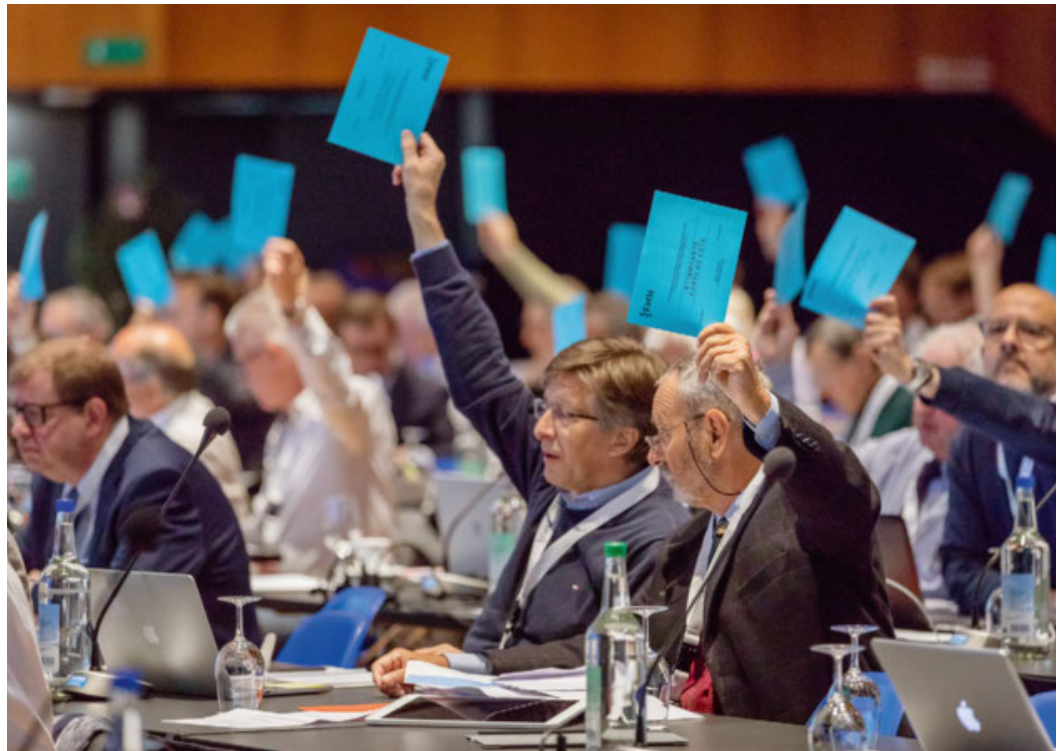

Die 200 Delegierten verabschieden einstimmig die Leistungsstruktur (Tarif-Nomenklatur) als wichtige Basis eines sachgerechten und betriebswirtschaftlich berechneten Tarifs.
2018 beim Bundesrat zur Genehmigung einzureichen. Neben dem zweiten Tarifeingriff per 1.1.2018 hat der Bundesrat am 28.3.2018 ein Kostendämpfungsprogramm verabschiedet. Eine international besetzte Expertengruppe hat 38 Vorschläge erarbeitet. Die Ärzteschaft wird Gelegenheit haben, zu den ersten Massnahmen, welche im Herbst 2018 in die Vernehmlassung geschickt werden, Stellung zu beziehen.

Trotz all dieser politischen Entscheide konnte der Zeitfahrplan eingehalten und die Meilensteine im Projekt TARCO erreicht werden. Die Delegierten haben an der a.o. DV vom 11.4.2018 die TARCO-Leistungsstruktur (Nomenklatur) einstimmig gutgeheissen. Die ÄK entscheidet heute ebenfalls über diese nachgebesserte Leistungsstruktur (Nomenklatur). Die definitiv bereinigte TARCO-Tarifstruktur inkl. Taxpunkte soll der DV am 27.6.2018 zur Genehmigung vorgelegt werden.

Urs Stoffel/ZV-Mitglied berichtet weiter, dass parallel zu den Projektarbeiten mit den Tarifpartnern $(\mathrm{H}+$, cufu, MTK und FMH) im Rahmen der ats-tms AG weiterverhandelt wird. Der ZV ist immer davon ausgegangen, eine bereinigte Tarifstruktur bis zum 31.3.2018 auszuarbeiten und diese bis Ende Juni 2018 den FMHinternen Gremien (DV und ÄK) vorzulegen. Am 2.2.2018 ist der VR der ats-tms in einem Mehrheitsentscheid zum Schluss gekommen, dass eine gemeinsame Einreichung einer unter den Tarifpartnern bereinigten Tarifstruktur bis 30.6.2018 unmöglich sei und neu nun per Ende 2018 vorgesehen ist.

Nach der Präsentation zum Stand des Projekts TARCO wird die Diskussion im Plenum eröffnet.
Auf die Fragen aus dem Plenum, ob eine Urabstimmung im Oktober 2018 vorgesehen ist, erklärt der Präsident, dass erste Diskussionen sowohl im ZV wie auch in der DV stattgefunden haben, jedoch keine Entscheide gefällt wurden. Um die Einreichungsfrist bis Ende 2018 nicht zu gefährden, wird der ZV von sich aus keinen Antrag stellen. Die ÄK hat jedoch gemäss Statuten entsprechende Möglichkeiten. Der Vorsitzende informiert noch über eine weitere Dimension, die es zu beachten gibt. Die Kommission für soziale Sicherheit und Gesundheit des Nationalrates (SGK-N) bezweckt mit der Parlamentarischen Initiative 17.401 Tarifpflege und Entwicklung eine Änderung von Art. 43 des KVG. In Abs. 5ter verlangt diese Kommissionsinitiative: «Die Tarifpartner setzen eine Organisation ein, die für die Erarbeitung und Weiterentwicklung sowie die Anpassung und Pflege der Tarifstrukturen zuständig ist ...» Die FMH hat alles Interesse, eine gemeinsame genehmigungsfähige Tarifstruktur fristgerecht einzureichen und sich für die Tarifautonomie einzusetzen. Dies entspricht auch dem Ziel 1 der verabschiedeten Legislaturzielen 2016-2020 der FMH. Aufgrund verschiedener längerer Voten verlangt Pierre Vallon/SGPP eine Redezeitbeschränkung.

\section{Ordnungsantrag Vallon:}

Redezeitbeschränkung auf drei Minuten.

\section{Beschluss:}

Der Antrag wird mit 150 Ja und 6 Nein genehmigt.

Nach weiteren Wortmeldungen orientiert Jürg Schlup/ Präsident FMH über den vom VSAO eingereichten Änderungsantrag zum Antrag 1 des ZV. Anja Zyska Cherix/ Präsidentin VSAO legt dar, dass ihr Berufsverband die vorgelegte Leistungsstruktur wie auch das vorgeschlagene Vorgehen unterstützen. Wie für den ZV sei es auch für den VSAO erklärtes Ziel, die Tarifautonomie $\mathrm{zu}$ behalten. Aus diesem Grund soll die vorliegende Leistungsstruktur (Nomenklatur) bei den weiteren $\mathrm{Ge}$ sprächen mit den Tarifpartnern nicht als endgültige Version, sondern als Grundlage für eine Konsensfindung verstanden werden. Sowohl gegenüber den in der ÄK vertretenen Organisationen, der Mitgliederbasis wie auch gegenüber den Verhandlungspartnern ist es wichtig, transparent zu machen, dass die von der FMH erarbeiteten Vorschläge im weiteren Dialog unter den Tarifpartnern noch Änderungen erfahren können. Diese Offenheit muss gewährleistet und klar kommuniziert sein, wenn man die Tarifpartnerschaft ernst nehmen und die Tarifautonomie bewahren bzw. zurückerlangen will.

Bei den nun folgenden Abstimmungen entscheiden die Delegierten als erstes, ob sie auf den Antrag VSAO eintreten wollen oder nicht. 
Antrag ZV:

Eintreten Ja oder Nein?

Beschluss:

Der Eintretensantrag wird mit 85 Ja, 30 Nein und 11 Enthaltungen angenommen.

Urs Stoffel/ZV-Mitglied und Departementsverantwortlicher Ambulante Versorgung und Tarife unterstützt den Änderungsantrag, wünscht jedoch, diesen mit der Klammerbemerkung (gemäss Tarifbrowser V2.2) zu ergänzen. Der VSAO ist damit einverstanden.

Pierre Vallon/Präsident $D V$ weist darauf hin, dass nicht nur das Cockpit, sondern auch die Delegierten an der a.o. DV vom 11.4.2018 die Leistungsstruktur verabschiedet haben. Er verlangt eine entsprechende Anpassung im Antrag des VSAO. Der VSAO ist mit dieser Präzisierung im Antrag einverstanden.

Jürg Schlup/Präsident FMH dankt dem Präsidenten der DV für diesen Hinweis. Er schlägt vor, zuerst über den unbestrittenen Antrag 2 des ZV und dann über die Anträge 1 des ZV und des VSAO abzustimmen.

Abstimmung 1 - Antrag 2 des ZV:

Die ÄK stimmt dem weiteren Vorgehen innerhalb des Projektes TARCO und den Verhandlungen mit den Tarifpartnern zu.

Beschluss:

Der Antrag wird mit 131 Ja, 0 Nein und 1 Enthaltung angenommen.

Abstimmung 2 - Antrag 1 des ZV:

Die ÄK stimmt der vorliegenden und vom Cockpit verabschiedeten Leistungsstruktur (Nomenklatur) und Kapitelstruktur des Projektes TARCO (gemäss Tarifbrowser V2.2) zu.

Beschluss:

Der Antrag wird mit 136 Ja, 0 Nein und 4 Enthaltungen angenommen.

Abstimmung 3 - Antrag VSAO:

Der VSAO beantragt, den ersten Passus des Antrags ZV wie folgt zu ergänzen:

Die ÄK stimmt der vorliegenden vom Cockpit und von der DV verabschiedeten Leistungsstruktur (Nomenklatur) und Kapitelstruktur des Projekts TARCO (gemäss Tarifbrowser V2.2) als Grundlage für die Verhandlungen mit den Tarifpartnern zu. Beschluss:

Der Antrag wird mit 115 Ja, 2 Nein und 14 Enthaltungen angenommen.

Urs Stoffel/ZV-Mitglied und Departementsverantwortlicher Ambulante Tarife und Versorgung bedankt sich bei den Delegierten, allen Mitwirkenden sowie seinem Team in Olten für den grossen Einsatz wie auch für das ihm ausgesprochene Vertrauen.
Jürg Schlup/Präsident FMH spricht vor allem Urs Stoffel, seinem Team wie auch allen Projektbeteiligten seinen Dank für das grosse Engagement aus.

\section{Donnerstag, 3. Mai 2018}

\section{Begrüssung, Mitteilungen, Bestellung des Büros}

Jürg Schlup/Präsident FMH begrüsst die Delegierten und alle eingeladenen Gäste zum zweiten Tag der Frühjahrs-ёK.

Nach den üblichen organisatorischen Mitteilungen durch Anne-Geneviève Bütikofer/Generalsekretärin FMH wird das Büro bestellt. Dieses besteht aus dem Präsidenten, dem Vizepräsidenten, der Generalsekretärin, Julien Duruz/RD FMH als Leiter Stimm- und Wahlbüro, Anne-Sylvie Thiébaud/RD FMH als stellvertretende Leiterin Stimm- und Wahlbüro, sowie den nachfolgenden einstimmig gewählten Stimmenzählenden:

Alexander Zimmer, Markus Bremgartner, Jean-Pierre Grillet, Florian Leupold, Paul Bösch, Franziska Zogg, Lucia Zürcher, Pierre Arnold, Karl-Olaf Lövblad, Marie-Claire Desax

\section{Genehmigung Traktandenliste}

Antrag:

Genehmigung der Traktandenliste.

Beschluss:

Die Traktandenliste wird mit 150 Ja, 1 Nein und ohne Enthaltung genehmigt.

Zu Beginn jeder Sitzung legt die ÄK gemäss Art. 11, Abs. 3 GO die Zeit fest, nach deren Ablauf weder Beschlüsse gefasst noch Wahlen vollzogen werden dürfen. Der Präsident schlägt als Tagungsende 17.00 Uhr vor.

Beschluss:

Der Antrag auf Festlegung des Tagungsendes auf 17.00 Uhr wird mit $150 \mathrm{Ja}, 0$ Nein und 1 Enthaltung angenommen.

Weiter stellt der Vorsitzende für die heutige ÄK zwei Ordnungsanträge. Gemäss Art. 11, Abs. 7 GO kann auf Vorschlag aus der Kammer oder des Vorsitzenden hin die Redezeit allgemein beschränkt werden. Aufgrund der Traktanden und der zur Verfügung stehenden Zeit wird eine Redezeitbeschränkung von zwei Minuten eingeführt.

Ordnungsantrag 1: Redezeitbeschränkung

Für die Behandlung der Traktanden der heutigen ÄK gilt eine Redezeitbeschränkung von 2 Minuten für Einzelredner. Keine Beschränkung gilt für 
den vom Präsidenten der jeweiligen stimmberechtigten oder antragsberechtigten Ärzteorganisation bezeichneten Sprecher und für den Sprecher des ZV.

Beschluss:

Der Antrag wird mit 150 Ja, 2 Nein und 9 Enthaltungen gutgeheissen.

Art. 11, Abs. 5 der GO sieht vor, dass jeder Delegierte, jedes ZV-Mitglied und der Generalsekretär das Recht haben, Anträge zu den zur Diskussion stehenden Traktanden zu stellen. Anträge sind dem Vorsitzenden schriftlich vor oder während der Sitzung oder ausnahmsweise mündlich einzureichen.

Ordnungsantrag 2: Schriftliche Einreichung der Anträge

Abänderungsvorschläge und Anträge sind dem Vorsitzenden schriftlich einzureichen. Mündliche Anträge werden nicht entgegengenommen und sind ungültig.

Beschluss:

Der Antrag wird mit 150 Ja, 2 Nein und 8 Enthaltungen gutgeheissen.

\section{Laudatio für den verstorbenen Pierre-Alain Schneider}

In dankbarer Erinnerung würdigt Jürg Schlup/Präsident FMH den am 24.3.2018 Verstorbenen. Mit Pierre-Alain Schneider verliert die Ärzteschaft einen engagierten Arzt, einen langjährigen Präsidenten mehrerer angeschlossener Ärzteorganisationen sowie den langjährigen und bis zu seinem Tod amtierenden Vizepräsidenten der FMH-Delegiertenversammlung. Für die Laudatio erteilt er Pierre Vallon/ Präsident DV das Wort.

Pierre Vallon/Präsident DV erinnert sich mit einfühlsamen Worten an einen bemerkenswerten Arztkollegen. Gleich einem Fels in der Brandung liess er sich weder von der Politik noch von den Versicherern beirren. Er beeindruckte immer wieder, auch nach langwierigen Debatten, mit seinen klaren und unmissverständlichen Statements. Nach seiner Pensionierung als Radiologe wollte er mehr Zeit für seine Familie verbringen und sich vermehrt seinen Hobbys, vor allem der Fliegerei, widmen. Seine Leidenschaft war die Fliegerei und es war sein Traum, nach der Pensionierung wiederum im Cockpit zu sitzen. Eine kurze und schwere Krankheit, welcher er mit viel Mut begegnet ist, hat diesen Traum jedoch viel zu früh beendet.

Die Anwesenden gedenken Pierre-Alain Schneider mit einer Schweigeminute.

\section{Eröffnungsreferat Präsident FMH}

Jürg Schlup/Präsident FMH erachtet eine gesundheitspolitische Strategieanpassung bei der Ärzteschaft als notwendig. Es braucht wieder eine stärkere Patientenorientierung. Die Ökonomie ist ein dienstbarer Geist; in der Medizin darf aber kein Primat der Ökonomie herrschen. Auch wenn aufgrund der steigenden $\mathrm{Ge}$ sundheitskosten wirtschaftliche Aspekte für die Ärztinnen und Ärzte eine grössere Bedeutung erhalten, hat die optimale Versorgung der Patienten stets Vorrang. Prof. Dr. Agnes Bäker zeigt in ihrer Studie «Why the best hospitals are managed by doctors» einen positive Zusammenhang von Qualität und Glaubwürdigkeit - bei allen Anspruchsgruppen wie Mitarbeitenden und Patienten - wenn Spitäler von einem Arzt geführt werden. Die empirische Evidenz zeigt tatsächlich, dass die besten Spitäler - gemäss den Rankings - von Ärzten geleitet werden. In Europa sind die Klinikdirektoren heute Ökonomen. Diese versuchen, Chefärzte für finanzielle Ziele zu instrumentalisieren und gefährden damit das Ansehen des Berufsstandes. Ein Primat der Ökonomie schadet der Qualität der Patientenversorgung und untergräbt das Vertrauen in das gesamte Versorgungssystem. Wenn das Vertrauen einmal verloren gegangen ist, wird es richtig teuer. In vielen Ländern Europas besteht ein Ärztemangel. Länder wie England investieren in den nächsten drei Jahren 100 Mio. Pfund für die Anwerbung von Hausärzten. In Deutschland vermeldet die Bundesärztekammer 2613 leer stehende Arztpraxen, die nicht wieder besetzt werden können. Und in der Schweiz haben sich gemäss Jobradar die ausgeschriebenen vakanten Arzt-Stellen in den letzten drei Jahren mehr als verdoppelt. Seit 2017 findet eine unausgewogene Berichterstattung zu den Ärzteeinkommen statt. Die vom Bundesamt für Gesundheit (BAG) in Auftrag gegebene und für Herbst 2017 angekündigte Einkommensstudie liegt immer noch nicht vor. Immerhin hat das Bundesamt für Statistik (BFS) im April 2018 die Ergebnisse der MAS-Strukturdaten publiziert. Das durchschnittliche Ergebnis der Einzelpraxis in der Schweiz liegt bei CHF 155000. Das im Frühjahr gefällte Urteil zum 1. Tarifeingriff 2014 hat die FMH mit Befremden zur Kenntnis genommen. Das Bundesgericht kommt zum Schluss, dass der Bundesrat die Tarifstruktur nicht länger nach sachgerechten und betriebswirtschaftlichen Kriterien gestalten, sondern sich auch von politischen Anliegen leiten lassen darf. Die Tarifpartner hingegen müssen sich weiterhin an die betriebswirtschaftliche Bemessung und Sachgerechtigkeit halten. Das Urteil hat Einfluss auf die laufenden Tarifverhandlungen und führt zu einer Rechtsunsicherheit. Die Weltwoche hat zum 
2. Tarifeingriff 2018 einen E-Mailaustausch zwischen dem Bundesamt für Justiz (BJ) und dem BAG publiziert. Darin erwähnt das BJ einen Generalvorbehalt zum Tarifeingriff 2018, dies in Bezug darauf, ob die gesetzlich vorgegebene Betriebswirtschaftlichkeit und Sachgerechtigkeit eingehalten werden. Die DatenschutzGrundverordnung der EU (DSGVO), welche Ende Mai 2018 in Kraft tritt, zwingt die Unternehmen in der Schweiz, bei der Bearbeitung von personenbezogenen Daten Sorgfalt walten zu lassen, um den Schutz der Privatsphäre jedes Einzelnen zu gewährleisten. Die Datenschutzbeauftragte der FMH hat an der Herbst-ÄK 2017 bereits darüber im Detail informiert. Der ZV hat Vorschläge zum weiteren Vorgehen gemacht, auf welche die ÄK damals nicht eingetreten ist. Das Generalsekretariat der FMH nimmt die Umsetzung der neuen Anforderungen des Datenschutzes sehr ernst und lässt sich - wie im Herbst 2017 von der ÄK gewünscht - von der gesetzlichen Pflicht leiten. Die wichtigsten politischen Herausforderungen 2018 für die Ärzteschaft bilden nach wie vor die Tarifrevision, die Umsetzung des Tarifeingriffs, die Verhinderung des Globalbudgets, die Mitgestaltung der Zulassungssteuerung, die einheitliche Finanzierung von ambulanten und stationären Leistungen sowie die Datenlieferungspflicht MAS-MARS. Der ZV erachtet vor allem die Tarifrevision, die Kostendämpfungsmassnahmen sowie die Zulassungssteuerung für Ärztinnen und Ärzte als wichtigste Herausforderung für die kommenden Monate.

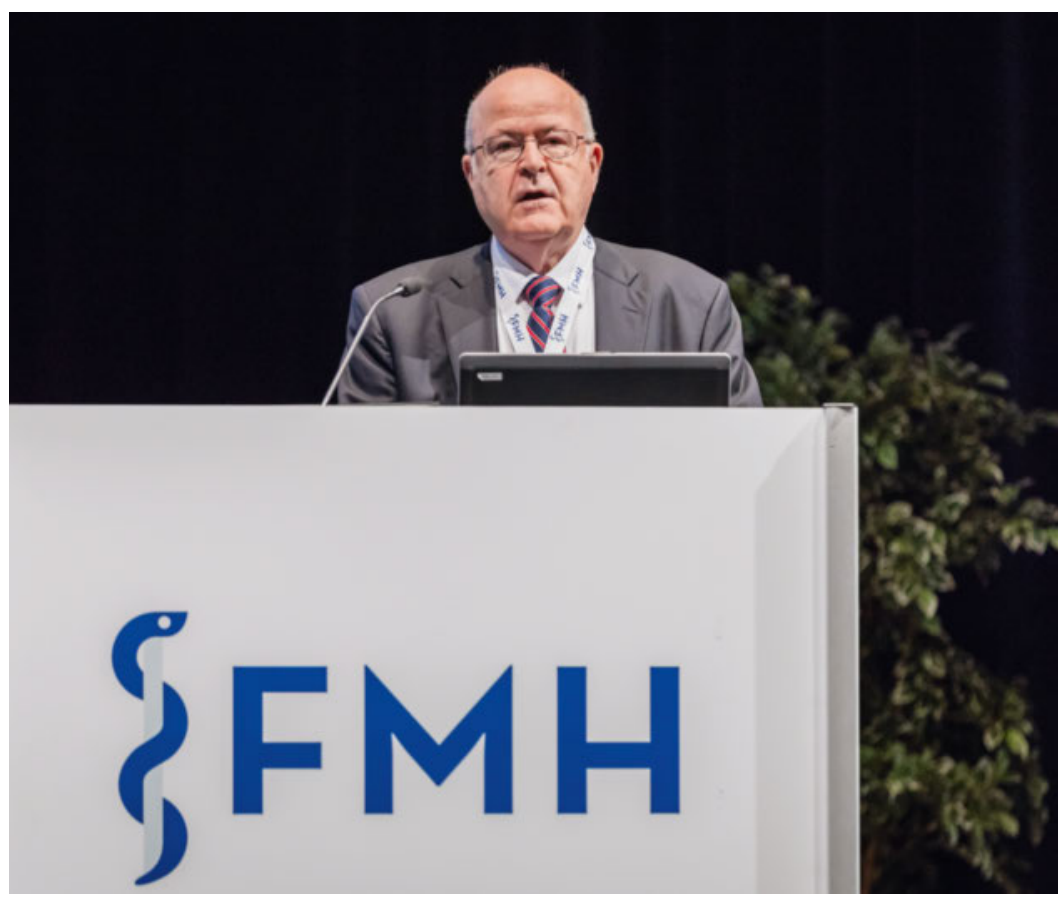

Werner Bauer, SIWF-Präsident, erläutert den Jahresbericht des SIWF 2017.

\section{Jahresberichte 2017}

\subsection{Jahresbericht 2017 des SIWF}

Werner Bauer/Präsident SIWF und Christoph Hänggeli/ Geschäftsführer SIWF berichten über die Aktivitäten und Schwerpunktprojekte des vergangenen Jahres. Bei der laufenden Akkreditierung, die der Bund alle sieben Jahre durchführt, sind die Grundlagen, die Selbstbeurteilung sowie die externe Begutachtung abgeschlossen. Der Entscheid des Eidgenössischen Departements des Innern (EDI) ist noch ausstehend. Grundsätzlich wird die Weiterbildung in der Schweiz von den internationalen und nationalen Experten positiv beurteilt. In der Plattform Ärztliche Bildung diskutiert zurzeit die Themengruppe «Wege und Strukturen der ärztlichen Bildung» die Frage, ob und wie die ärztliche Bildung zeitlich verkürzt werden soll. Wie sich eine Verkürzung auf die Gesundheitsversorgung und die Qualität auswirken wird, ist offen. Die Frage nach der Effizienz der Weiterbildung ist berechtigt und gerät auch vermehrt in den Fokus. Auch zum Thema Fortbildung will sich das BAG vermehrt engagieren. Es regt die Bildung einer entsprechenden Themengruppe an, welche einen allfälligen Regulierungsbedarf prüfen soll. Die Entrustable Professional Activities (EPA) kommen aus der kompetenzbasierten Bildung und sind ein Kompetenzset, das man erlernen und evaluieren muss. Im neuen Katalog der Lernziele für das Studium in der Schweiz sind solche EPAs bereits eingebaut worden. Das SIWF wird sich in der Weiterbildung zusammen mit einer Arbeitsgruppe intensiver damit befassen. Das jährliche Medical Education Symposium findet am 12.9.2018 in Bern statt.

Christoph Hänggeli/Geschäftsführer SIWF ist überzeugt, dass mit der am 1.1.2018 in Kraft gesetzten Revision des Medizinalberufegesetzes (MedBG) zwei wichtige Anliegen für den Patientenschutz realisiert werden konnten. Einerseits müssen alle Ärztinnen und Ärzte vor Aufnahme einer beruflichen Tätigkeit ihr Diplom überprüfen und sich im offiziellen Medizinalberuferegister (MedReg) eintragen lassen. Andererseits müssen die für die jeweilige Berufsausübung notwendigen Sprachkenntnisse nachgewiesen werden. Ein Wermutstropfen bleibt: Die mit einer Schweizer Matura ausgewiesenen Sprachen werden nicht anerkannt und können im Register nicht eingetragen werden. Die Neuentwicklung des e-Logbuches 2.0 läuft plangemäss. Der vom SIWF beauftragte externe Experte versichert, dass die neue, modular aufgebaute Version über eine besser strukturierte Programmierung verfügt, die auch künftigen Anforderungen genügen wird. Aus seiner Sicht sind keine strategischen Interventionen angezeigt. Der Investitionsbedarf für 2018 und 
2019 beläuft sich auf jeweils CHF 1 Mio. Die e-Fortbildungsplattform funktioniert als Tool ohne technische Probleme. Damit können die Fachgesellschaften die Fortbildungskontrolle für alle Anträge einfach erledigen. Aktuell sind erst 50\% der Ärzte im Besitz eines Fortbildungsdiploms (Österreich: 95\%). Dieser niedrige Wert wird vom Bund als Argument verwendet, im Fortbildungsbereich regulieren zu wollen. Die Erteilung der Facharzttitel ist nach dem Einbruch im Jahr 2016 wieder auf Niveau von 2013. Die Gesuchzunahme ist erfreulich und wird sich positiv auf die Rechnung 2018 auswirken.

Weitere detaillierte Informationen zum Jahresbericht sind elektronisch unter www.fmh.ch/bildung-siwf/ ueber-siwf/geschaeftsberichte.html abrufbar.

Antrag SIWF:

Die Geschäftsleitung SIWF beantragt der ÄK, den Jahresbericht 2017 des SIWF zu genehmigen. Beschluss:

Der Jahresbericht des SIWF wird einstimmig genehmigt.

\subsection{Jahresbericht 2017 der FMH}

Gemäss Anne-Geneviève Bütikofer/Generalsekretärin $F M H$ entspricht die e-Version den heutigen technischen Anforderungen und garantiert einen optimierten $\mathrm{Zu}$ griff für alle zur Verfügung stehenden Endgeräte. Wie der Präsident bereits in seinem Eröffnungsreferat darlegte, ist die aktuelle politische Situation im Gesundheitswesen für die FMH nach wie vor eine grosse Herausforderung. Das Generalsekretariat hat sich im

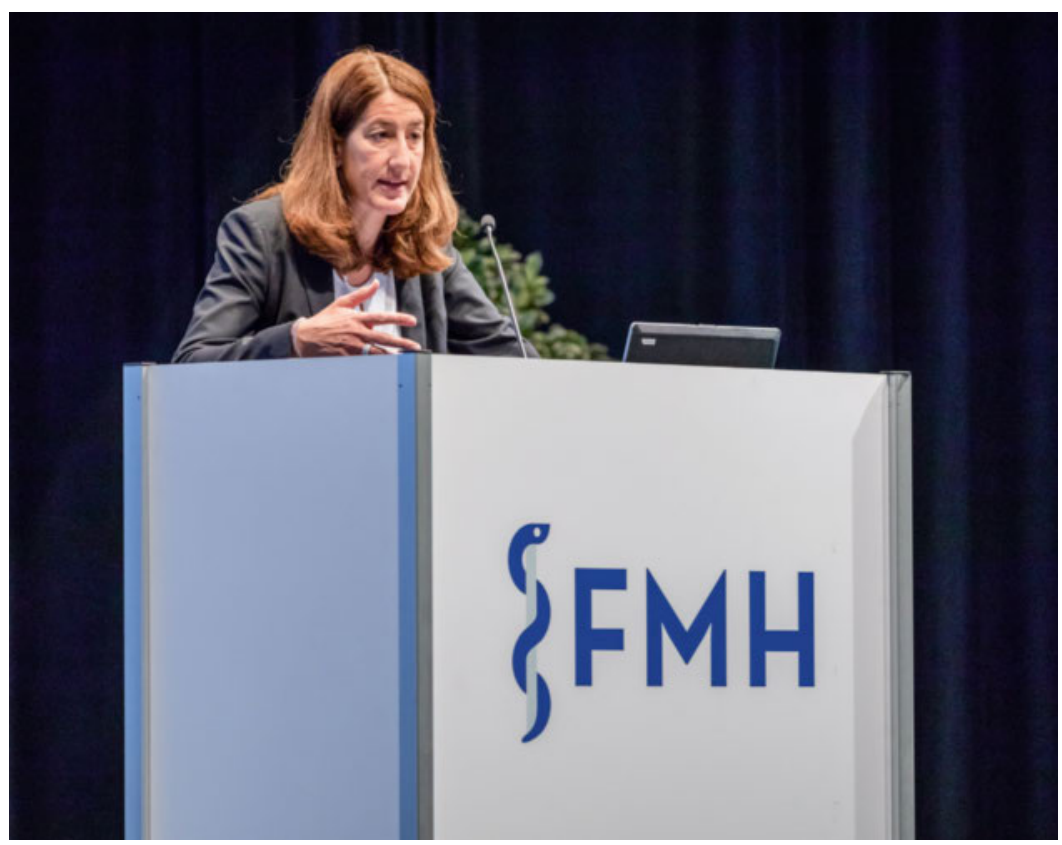

Die Generalsekretärin Anne-Geneviève Bütikofer berichtet über die Budgetstabilisierungsmassnahmen. vergangenen Jahr intensiv mit dem Finanzhaushalt und der Erreichung eines finanziellen Gleichgewichts zwischen Ausgaben und Einnahmen beschäftigt. Die für 2017 definierten Ziele für die Umsetzung der Budgetstabilisierungsmassnahmen wurden übertroffen. Die Analyse der Geschäftsprozesse, die Umsetzung des Risikomanagements wie auch die Erarbeitung einer ICT-Strategie sind weitere erwähnenswerte Aktivitäten. Das strategische Projekt I-Governance ist in Verzug. Beim Rechtsdienst wurde das Reglement der Gutachterstelle wie auch die dritte Edition des Guide pratique FMH/ASSM überarbeitet. Die Revision der Weiterbildungsverordnung MPA war ressourcenintensiv und kann 2018 abgeschlossen werden. Das Projekt TARCO, der Dienstleistungskatalog wie auch ein grösseres PR für die Mitglieder der FMH bedingten eine grosse Kommunikationsarbeit. Die Mitgliederzahlen sind 2017 erfreulicherweise wiederum auf 42301 Mitglieder gestiegen.

Im letzten Jahr wurde der Fokus auf die Kulturentwicklung der FMH gelegt. Führungskräfte und Mitarbeitende setzten sich im Rahmen verschiedener Seminare und Workshops mit den Werten der FMH auseinander. Im Wissen, dass die Arbeitskultur kein statisches Konzept ist, wird dieser Prozess auch in den kommenden Jahren weitergeführt. Per 31.12.2017 betrug der Personalbestand 102 Mitarbeitende (davon 76 für das Generalsekretariat) und hat sich im Vergleich zum Vorjahr leicht reduziert. Das Durchschnittsalter lag bei 48 Jahren. Die durchschnittliche Betriebszugehörigkeit betrug 9 Jahre, wobei 14 Mitarbeitende bereits 20 oder mehr Dienstjahre geleistet haben. Die Geschlechterverteilung innerhalb der FMH widerspiegelt eine Mehrheit der Frauen. 2017 waren 68 Frauen (67\%) und 34 Männer (33\%) angestellt. Der Anteil der Frauen in leitenden Positionen liegt bei 39\%. Die Mitarbeiterfluktuation (netto) betrug 10,7\%. Die Anzahl Eintritte stieg auf 14 Personen (Vorjahr 9 Personen).

Weitere detaillierte Informationen des Jahresberichts sind unter www.report2017.fmh.ch abrufbar.

\section{Antrag ZV:}

Der ZV FMH beantragt der ÄK, den Jahresbericht 2017 der FMH zu genehmigen.

\section{Beschluss:}

Der Jahresbericht der FMH wird einstimmig genehmigt.

\section{Bericht 2017 Budgetstabilisierungs- massnahmen}

Anne-Geneviève Bütikofer/Generalsekretärin FMH rapportiert über die Zielerreichung der für 2017 umzusetzenden Massnahmen. Ziel der ÄK war die Schaf- 
fung eines finanziellen Gleichgewichts zwischen Ausgaben und Einnahmen. Die Einsparungen von total CHF 5,8 Mio. sollten in zwei Etappen erreicht werden, einerseits durch Aufwandsverminderungen und andererseits durch Ertragssteigerungen. Die an der ÄK vom 28.10.2015 beschlossenen 116 Budgetstabilisierungsmassnahmen werden seit Frühjahr 2016 mit einem eigens dafür entwickelten Evaluationsprozess laufend überprüft. Nach Abschluss des Geschäftsjahrs 2017 resultiert ein positives Resultat in der Höhe von TCHF 377 bzw. 39\%. Der SOLL-Betrag liegt bei TCHF 965 und der IST-Betrag bei TCHF 1342. Die Hauptdifferenzen sind auf Mehreinnahmen bei den Mitgliederbeiträgen, auf die Reduktion der Arbeitgeberbeiträge an die 2 . Säule sowie auf einen Minderaufwand bei Rechtsberatungen von Mitgliedern zurückzuführen. Für die Jahre 2018 und 2019 sind noch weitere Einsparungen von CHF 1 Mio. zu realisieren. Die ÄK wird am 25.10.2018 über die Zielerreichung des ersten Semesters 2018 informiert.

Die ÄK-Delegierten nehmen den Stand der Umsetzungsarbeiten zu den Budgetstabilisierungsmassnahmen aus dem Geschäftsjahr 2017 zur Kenntnis.

\section{Jahresrechnung 2017}

\subsection{Jahresrechnung 2017 des SIWF}

Christoph Hänggeli/Geschäftsführer SIWF erläutert die Gründe, die zum besseren Ergebnis geführt haben. Nach einem budgetierten Verlust von TCHF 867 schliesst die Jahresrechnung mit einem Minus von TCHF $645 \mathrm{ab}$. Die Abweichungen zwischen Budget und Rechnung sind ertrags- und aufwandseitig begründet. Im Vergleich zu 2016 sind die Mehreinnahmen von fast einer Million dank den Zertifizierungsgebühren und der höheren Zahl erteilter Facharzttitel zustande gekommen. Der Mehraufwand gegenüber 2016 ist vor allem durch das e-Logbuch, die Akkreditierungskosten wie auch durch Mindereinnahmen bei den Fortbildungsdiplomen begründet. Das SIWF versucht jeweils, die nach Kostenstellen erfassten Aufgabenbereiche kostendeckend zu finanzieren. Im Bereich der Weiterbildungsstätten beträgt der Kostendeckungsgrad inzwischen fast $100 \%$. Dieses Ziel ist jedoch in anderen Bereichen wie z.B. bei den Einsprachen nicht zu erreichen. Der Justizbereich muss auch beim Staat aus allgemeinen Steuermitteln finanziert werden. Christoph Hänggeli gibt einen Ausblick auf die Zahlen des ersten Quartals 2018, welche positiv sind. Mit den bereits beschlossenen Massnahmen wird das SIWF das Budget voraussichtlich im Jahr 2020 konsolidiert haben. Der Vermögensstand des SIWF beträgt per 1.1.2018 CHF 2,790 Mio.

\subsection{Jahresrechnung 2017 der FMH}

Emanuel Waeber/Leiter Abteilung Verwaltung und Finanzen präsentiert die Jahresrechnung der FMH, welche mit einem Gewinn von TCHF 687 abschliesst. Die wesentlichen Ertragsabweichungen sind auf die $\mathrm{Zu}$ nahme der Mitgliederbeiträge sowie auf Dienstleistungs- und Finanzerträge zurückzuführen. Beim Aufwand sind sowohl die Projekt-, die Personal- wie auch die Verwaltungs- und Informatikkosten tiefer. Weiter trägt die Umsetzung der Budgetstabilisierungsmassnahmen wesentlich zum positiven Ergebnis bei.

Die FMH hat 2017 erstmals die Bilanzsumme von CHF 28 Mio. überschritten. Mit CHF 15,132 Mio. sind die flüssigen Mittel gegenüber dem Vorjahr um TCHF 700 gestiegen. Das Anlagevermögen bleibt mit CHF 6,764 Mio. unverändert. Das kurzfristige Fremdkapital ist gegenüber dem Vorjahr aufgrund des verminderten Projektaufwands um knapp CHF 2 Mio. tiefer, das langfristige Fremdkapital aufgrund vorgenommener Rückstellungen um CHF 2,56 Mio. höher. Das Eigenkapital beträgt CHF 13,6 Mio.; die Anlageperformance $6,46 \%$.

Die konsolidierte Jahresrechnung schliesst mit einem Gewinn von TCHF 42 ab. Budgetiert war ein Verlust von TCHF 217. Der ZV schlägt folgende Verwendung des Jahresergebnisses vor: Zuweisung an das Kapital der FMH TCHF 687; Zuweisung an die Reserve des SIWF - TCHF 645.

\section{Bericht der Revisionsstelle BDO}

Simon Kehrli/Revisionsstelle BDO bestätigt die Prüfung des Jahresrechnung 2017 durch die Revisionsstelle. Nach ihrer Beurteilung entspricht diese für das am 31.12.2017 abgeschlossenen Geschäftsjahr dem schweizerischen Gesetz und den Statuten. Die Revisionsstelle bestätigt in Übereinstimmung mit Art. 728 a, Abs. 1 Ziffer 3 OR und dem Schweizerischen Prüfungsstandard 890, dass ein gemäss den Vorgaben des ZV ausgestaltetes internes Kontrollsystem für die Aufstellung der Jahresrechnung existiert. Die Revisionsstelle empfiehlt, die Jahresrechnung 2017 zu genehmigen.

\subsection{Bericht der Geschäftsprüfungskommission (GPK) FMH}

Adrian Sury/Präsident GPK berichtet über die Sitzungstätigkeit, welche im vergangenen Jahr vor allem der Überwachung der von der ÄK im Herbst 2015 verabschiedeten Budgetstabilisierungsmassnahmen, dem Risiko- und Prozessmanagement, den Datenschutzvorschriften, laufenden Projekten wie Fakturierung Zentralbeiträge, Website, e-Logbuch und Gesamtsanierung gewidmet war. Die Kommission hat in der gleichen Zusammensetzung getagt. Jean-Claude Brückner 
hat jedoch auf Ende April 2018 seinen Rücktritt bekanntgegeben.

Die gemeinsamen halbjährlichen Sitzungen mit der FMH und dem SIWF waren stets wertschätzend und konstruktiv. Die GPK hat die Jahresrechnung 2017 geprüft und beantragt der ÄK die Genehmigung sowie die Décharge-Erteilung für den ZV und den Vorstand SIWF.

Adrian Sury/Präsident GPK dankt den Präsidenten der FMH und des SIWF, dem ZV, dem Generalsekretariat sowie der Geschäftsstelle SIWF für die ausgezeichnete Zusammenarbeit.

\subsection{Genehmigung der konsolidierten Erfolgs-} rechnung 2017

Antrag ZV:

Der ZV FMH beantragt der ÄK, die Erfolgsrechnung $2017 \mathrm{zu}$ genehmigen.

Beschluss:

Die Erfolgsrechnung 2017 wird mit 138 Ja, ohne Gegenstimmen und 2 Enthaltungen genehmigt.

\section{Décharge-Erteilungen}

Der Vorstand SIWF und der ZV FMH beantragen der ÄK, den Empfehlungen der GPK sowie der Kontrollstelle zu folgen und ihnen für das Geschäftsjahr 2017 Décharge zu erteilen.

\subsection{Décharge-Erteilung SIWF}

Antrag SIWF:

Der Vorstand SIWF beantragt der ÄK, den Empfehlungen der Kontrollstelle sowie der GPK zu folgen und dem Vorstand SIWF für das Geschäftsjahr 2017 Décharge zu erteilen.

Beschluss:

Die ÄK erteilt dem Vorstand SIWF mit grossem Mehr, ohne Gegenstimmen und 4 Enthaltungen Décharge für das Geschäftsjahr 2017.

\subsection{Décharge-Erteilung Zentralvorstand FMH Antrag ZV:}

Der ZV FMH beantragt der ÄK, den Empfehlungen der Kontrollstelle sowie der GPK zu folgen und dem ZV FMH für das Geschäftsjahr 2017 Décharge zu erteilen.

Beschluss:

Die ÄK erteilt dem ZV FMH mit grossem Mehr, ohne Gegenstimmen und 3 Enthaltungen Décharge für das Geschäftsjahr 2017.

\section{Wahlen und Bestätigungswahlen}

6.1 Wahl 2. Vizepräsidium ZV FMH (vakant) Die ÄK hat am 26.10.2017 anlässlich der Ersatzwahlen Michel Alexandre Matter neu in den Vorstand gewählt. Die Wahl für das 2. Vizepräsidium wurde damals auf

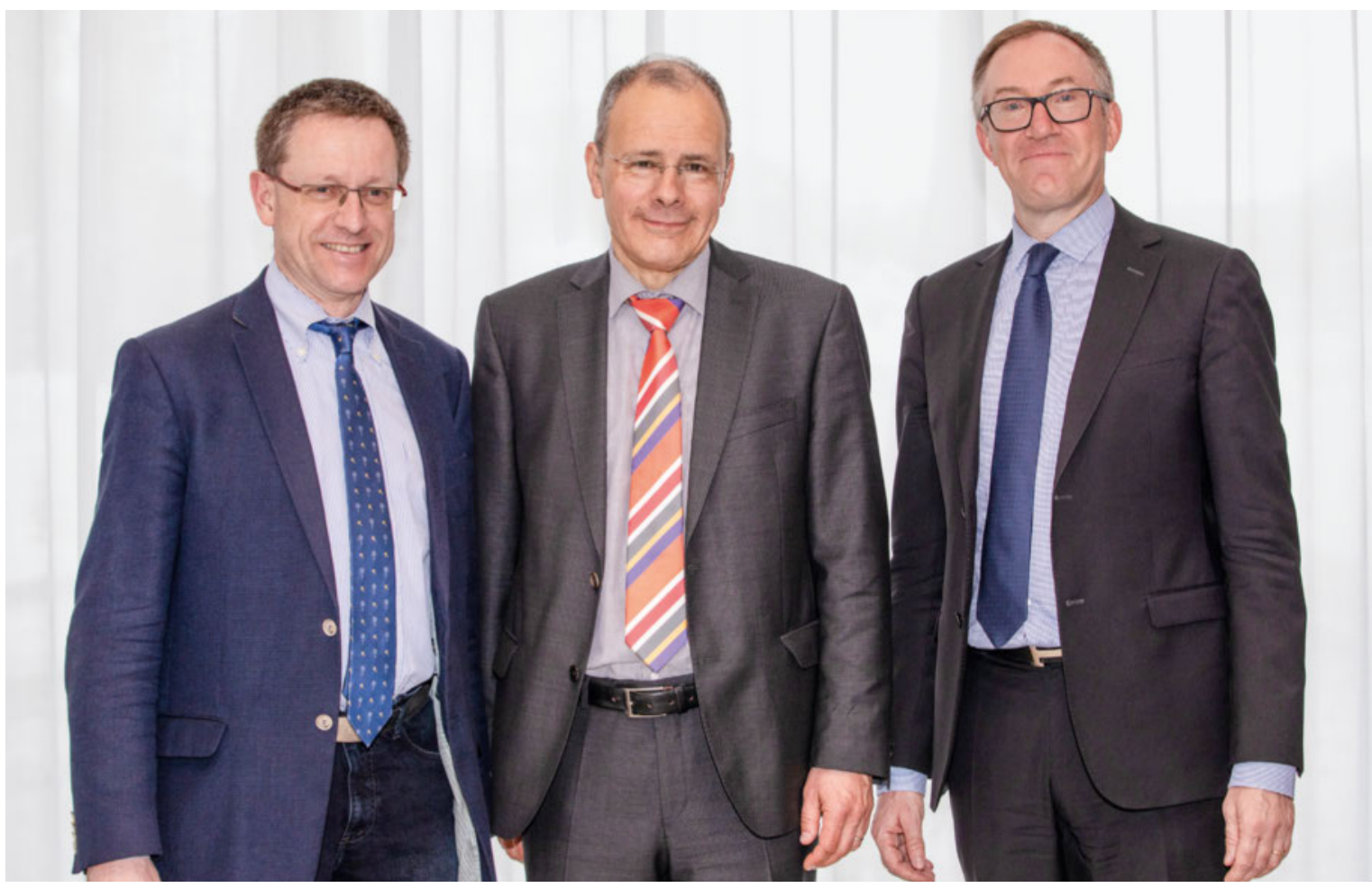

Das FMH-Präsidium ist mit der Wahl von Michel Matter wiederum komplett. V.I.n.r: Christoph Bosshard (Vizepräsident), Jürg Schlup (Präsident) und Michel Matter (Vizepräsident). 
die Frühjahrs-ÄK 2018 verschoben. Für die heutige Ersatzwahl für das 2. Vizepräsidium stellt sich Michel Alexandre Matter zur Verfügung.

Felix Kurth/VSAO unterstützt grundsätzlich diese Kandidatur. Der VSAO bedauert jedoch, dass durch diese Kandidatur keine Frau im Präsidium der FMH vertreten ist. Dadurch zementiert sich unglücklicherweise die ungenügende Vertretung von Ärztinnen in den wichtigen Organen der FMH. Der Frauenanteil in der Ärzteschaft beträgt heute über $40 \%$. Es ist absolut notwendig, den Frauenanteil in den Gremien der FMH künftig massiv zu erhöhen. Dies soll ein Appel nicht nur an die Ärztinnen sein, sich zu bewerben, sondern auch an alle Männer, Frauenkandidaturen zu ermöglichen.

Jürg Schlup/Präsident FMH dankt für dieses Votum und appelliert an alle in der ÄK angeschlossenen Organisationen, Frauenkandidaturen zu unterstützen und zu fördern.

Im ersten Wahlgang erhalten Michel Alexandre Matter 120, Yvonne Gilli 11 und Urs Stoffel 5 Stimmen. Michel Alexandre Matter wird mit 120 Stimmen zum 2. Vizepräsidenten der FMH gewählt.

Der Präsident gratuliert dem neuen Vizepräsidenten herzlich zur Wahl. Michel Alexandre Matter seinerseits bedankt sich für das ausgesprochene Vertrauen.

\subsection{Ersatzwahl Mitglied GPK (Ersatz Jean-Claude Brückner)}

Jean-Claude Brückner wurde 2012 als Vertreter der Romandie in die GPK gewählt. Er hat per Ende April 2018 seine Demission eingereicht. Adrian Sury/Präsident GPK dankt dem scheidenden Mitglied für sein ausserordentliches und grosses Engagement. Seine langjährige berufliche und persönliche Erfahrung sowie seine analytische Denkweise haben die Kommission in der Ausübung ihrer Aufgaben bereichernd unterstützt. Hervorzuheben sind seine differenzierten und kritischen Fragen, welche wichtig waren, die Problemstellung auch aus einem anderen Blickwinkel zu betrachten. Adrian Sury/Präsident GPK wünscht Jean-Claude Brückner eine gute Gesundheit, viel Zeit und Freude für seine Hobbies.

Jürg Schlup/Präsident FMH schliesst sich diesem Dank an und wünscht dem abtretenden Mitglied der GPK für die Zukunft alles Gute.

Für die Ersatzwahl innerhalb der gesetzten Frist hat einzig die Waadtländer Ärztegesellschaft SVM die Kandidatur von Philippe Vuillemin eingereicht. Da es keine weiteren Kandidaturen gibt, präsentiert Philippe Eggimann/Präsident SVM die zur Wahl stehende Persönlichkeit. Er bezeichnet Philippe Vuillemin als einen Kollegen mit einer jahrelangen beruflichen und politischen Erfahrung. Als GPK-Mitglied und Vizepräsident des Grossen Rates des Kantons Waadt wie auch als Mitglied der Geschäftsprüfungskommission der Stadt Lausanne und weiteren Mandaten konnte er sich vertiefte Verwaltungs- und Managementerfahrungen aneignen. Philippe Vuillemin ist ein äusserst qualifizierter Kandidat und bringt alle erforderlichen Kompetenzen für dieses Amt mit sich. Er empfiehlt den Delegierten, die Kandidatur zu unterstützen.

Philippe Vuillemin wird im ersten Wahlgang - bei 146 eingegangenen Stimmzetteln - mit 135 Stimmen zum neuen Mitglied der GPK gewählt.

Jürg Schlup/Präsident FMH gratuliert dem neuen GPKMitglied und wünscht ihm in dieser Funktion viel Erfolg und Befriedigung.

\subsection{Wiederwahl der Kontrollstelle FMH}

Jürg Schlup/Präsident FMH schlägt vor, die Wiederwahl der Kontrollstelle offen durchzuführen.

Antrag:

Die Wiederwahl der Kontrollstelle wird als offene Wahl durchgeführt.

\section{Beschluss:}

Der Antrag wird mit 130 Ja, 1 Nein und ohne Enthaltung angenommen.

Antrag ZV:

Der ÄK wird beantragt, für die Jahre 2019 und 2020 die Firma BDO AG in Bern als Kontrollstelle zur Wiederwahl vorzuschlagen. Die GPK schliesst sich diesem Antrag des ZV an.

Beschluss:

Die Kontrollstelle wird mit 143 Ja, ohne Gegenstimme und ohne Enthaltung für die Jahre 2019 und 2020 wiedergewählt.

\subsection{Bestätigungswahl von nominierten DV-Delegierten}

Art. 36a der FMH-Statuten sieht vor, dass die Delegierten der DV durch die ÄK bestätigt werden. Die zu bestätigenden Delegierten sind ausschliesslich stimmoder antragsberechtigte ÄK-Delegierte.

\section{Antrag VSAO und FMCH:}

Die antragsstellende Organisationen VSAO und FMCH beantragen der ÄK, folgende Delegierte neu ab dem 3.5.2018 für die Delegiertenversammlung der FMH zu bestätigen:

\section{VSAO}

Bisher: Christoph FEHLMANN, Cologny

Neu: Maryline FOERSTER PIDOUX, Lausanne

Bisher: Daniel SCHRÖPFER, Oberengstringen

Neu: Marius GRÄDEL-SUTER, Bern

Bisher: Urs Sascha SIEBER, Liebefeld

Neu: $\quad$ Aleksandra POROWSKA, Lausanne 


\section{FMHC}

Bisher: vakant

Neu: Urs KAUFMANN, Wabern

Beschluss:

Die Delegierten werden einstimmig bestätigt.

\subsection{Bestätigungswahl von nominierten DV-Ersatzdelegierten}

Art. 36a der FMH-Statuten sieht vor, dass die Ersatzdelegierten der DV durch die ÄK bestätigt werden. Die zu bestätigenden Ersatzdelegierten sind ausschliesslich stimm- oder antragsberechtigte ÄK-Delegierte.

Antrag VLSS, mws, VSAO:

Die antragsstellende Organisationen VLSS, mws und VSAO beantragen der ÄK, folgende Ersatzdelegierte neu ab dem 3.5.2018 für die Delegiertenversammlung der FMH zu bestätigen: VLSS

Bisher: vakant

Neu: Michaela PETRE, Winterthur

mws

Bisher: vakant

Neu: Susanna STÖHR, Basel

VSAO

Bisher: Lars FRAUCHIGER, Innerberg

Neu: Michel CLÉMENT, Bern
Beschluss:

Die Ersatzdelegierten werden einstimmig bestätigt.

\section{7. Änderungen in Statuten, Geschäfts- ordnung und Standesordnung}

\section{1 Änderung in der Standesordnung FMH}

\subsection{1 Übernahme der SAMW-Richtlinie «Betreuung} und Behandlung von Menschen mit Demenz"

Die SAMW hat die Richtlinie im letzten Herbst verabschiedet. Jürg Schlup/Präsident FMH begrüsst PD Dr. med. Georg Bosshard/Zürich, der die Subkommission innerhalb der Zentralen Ethikkommission der SAMW geleitet hat. Der Referent informiert über die wesentlichen Ziele und Themenfelder der vom Bund und von den Kantonen 2014 lancierten Nationalen Demenzstrategie. Ziel 5 dieser Strategie legt fest, die Lebensqualität dieser wachsenden Patientengruppe zu verbessern, Belastungen zu verringern und die Qualität der Versorgung zu garantieren. In der Schweiz leben rund 120000 Menschen mit Demenz.

Die SAMW hat jahrelange Erfahrung im Etablieren von medizinisch-ethischen Richtlinien. Es war auch ihr Ziel, mit dieser Richtlinie für die Betreuung und Behandlung von Menschen mit Demenz eine praktische

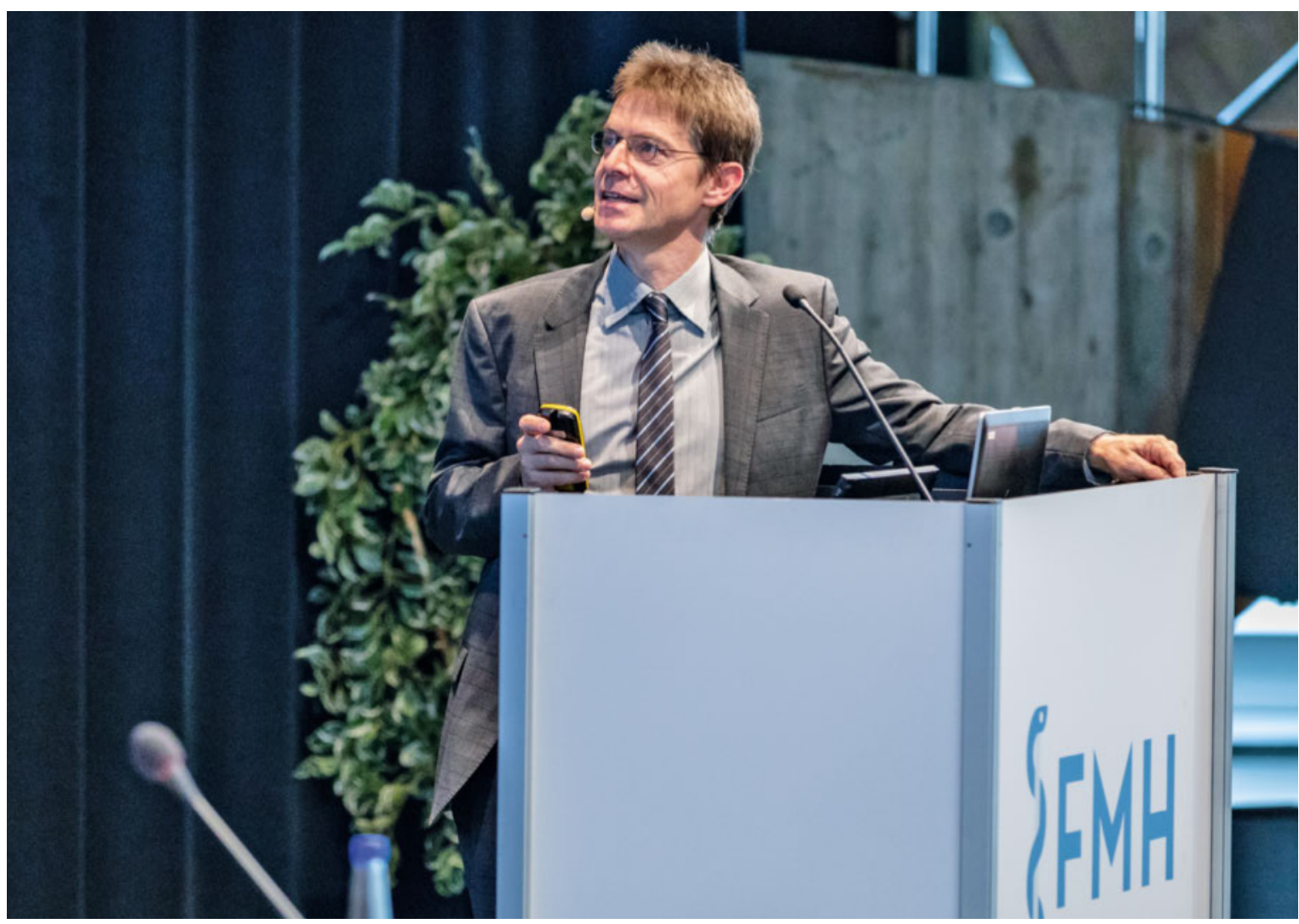

PD Dr. med. Georg Bosshard spricht über die SAMW-Richtlinien «Betreuung und Behandlung von Menschen mit Demenz». 
Orientierungshilfe in ethischen Konfliktsituationen zu bieten. Dabei wird die Gesamtthematik einzelnen Problemfeldern zugeordnet, wie sie sich letztlich setting-übergreifend (zu Hause, im Spital, im Pflegeheim) wie auch berufsgruppenübergreifend allen Betreuungspersonen stellen können. Bei den Grundsätzen wurden Würde, Lebensqualität, Wohlbefinden, Wahrhaftigkeit und Respekt als relevant erachtet. Demenz ist jene Krankheit in der Medizin, wo die Zusammenarbeit mit den Angehörigen vielleicht den wichtigsten Stellenwert überhaupt hat. Häufig ist es ja so, dass die Angehörigen an der Demenzerkrankung fast mehr leiden als die betroffene Person selber. Es wird auch auf die Frage mit dem Umgang eines Suizidwunsches eingegangen. Die SAMW hat diesbezüglich eine weitere Richtlinie in Vernehmlassung, die sich vertiefter mit dieser Thematik auseinandersetzt.

Nach verschiedenen Voten aus dem Plenum zum Suizidwunsch unterstreicht Jürg Schlup/Präsident FMH, dass die zu verabschiedende Richtlinie einen gewissen Zusammenhang zu der aktuell in Vernehmlassung stehenden SAMW-Richtlinie "Umgang mit Sterben und Tod» hat. Die Verabschiedung dieser Richtlinie ist jedoch erst für die nächste ÄK vorgesehen.

\section{Antrag ZV:}

Der ZV beantragt die Übernahme der Richtlinie «Betreuung und Behandlung von Menschen mit Demenz" in die Standesordnung

\section{Beschluss:}

Der Antrag wird mit 128 Ja, 1 Nein und 13 Enthaltungen angenommen.

\subsubsection{Vororientierung SAMW-Richtlinie "Umgang mit Sterben und Tod»}

Wie vorgängig erwähnt, revidiert die SAMW zurzeit die Richtlinie «Betreuung von Patientinnen und $\mathrm{Pa}$ tienten am Lebensende». Unter dem neuen Titel «Umgang mit Sterben und Tod» wurde ein Entwurf bereits vernehmlasst. In dieser neuen Richtlinie steht das Gespräch über Sterben und Tod im Zentrum. Dr. iur. Michael Barnikol/RD FMH beschränkt sich in seinen Darlegungen auf denjenigen Punkt, der umstritten ist und der auch Anlass für diese Vororientierung gegeben hat. Das ist die Regelung der Suizidbeihilfe. Die neue Regelung ist ähnlich strukturiert wie die alte. Suizidbeihilfe ist nach beiden Regelungen keine Verpflichtung des Arztes, entsprechend gibt es keinen Anspruch des Patienten auf Beihilfe zum Suizid. Beide Richtlinien sehen vor, dass dann, wenn ein Arzt sich dazu entschliesst, Beihilfe zum Suizid zu leisten, er vorgängig bestimmte Voraussetzungen prüfen muss hierzu gehört vor allem die Urteilsfähigkeit des Patienten. Suizidbeihilfe darf er nur dann leisten, wenn diese Voraussetzungen gegeben sind. Bei der geltenden Regelung trägt der Arzt, der sich zur Beihilfe zum Suizid entschliesst, insbesondere die Verantwortung für die Prüfung der folgenden Voraussetzung: Die Erkrankung des Patienten rechtfertigt die Annahme, dass das Lebensende nahe ist. Alternative Möglichkeiten der Hilfestellung wurden erörtert und soweit gewünscht auch eingesetzt. Bei der neuen Regelung kann ein Arzt aufgrund eines persönlich verantworteten Entscheides insbesondere nur dann Suizidhilfe leisten, wenn die Krankheitssymptome und/oder Funktionseinschränkungen des Patienten für diesen Ursache unerträglichen Leidens sind. Der Begriff «unerträgliches Leiden» ist unbestimmt und für den betroffenen Arzt schwer feststellbar. Es wird vom Arzt verlangt, ein Urteil darüber zu fällen, was für den Patienten (noch) erträglich ist. Die Regelung ist problematisch bei Patienten, die aufgrund einer psychischen Erkrankung suizidgefährdet sind. Diese Regelung betrifft nicht mehr die ursprüngliche Zielsetzung der Richtlinie, den Umgang mit sterbenden und todkranken Patienten zu regeln.

Die Bedeutung der neuen Richtlinie ist gross, da sie nach Genehmigung durch die ÄK Teil der Standesordnung der FMH ist. Die Delegierten werden an der Herbst-ÄK über die Aufnahme dieser Richtlinie in die Standesordnung der FMH beschliessen. Der ZV hat in seiner Stellungnahme an die SAMW darauf hingewiesen, dass die Suizidhilfe sich auf jene Patienten beschränken sollte, die an einer tödlichen Krankheit leiden und deren Zustand sich bei einer sachgerechten medizinischen Behandlung auch nicht bessern wird. Eine solche Diagnose kann ein Arzt mit hinreichender Zuverlässigkeit stellen.

Ob die SAMW die Bedenken der FMH in ihre neue Richtlinie aufnehmen wird, ist nicht bekannt. Einzelne Votanten regen an, nochmals mit der SAMW Gespräche zu führen und den Standpunkt der Ärzteschaft darzulegen. Der Vorsitzende prüft das Anliegen der Votanten.

\section{2 Änderung in den Statuten FMH}

\subsubsection{Antrag mfe: Aufnahme mfe als mitsprache-} berechtigte Organisation der FMH

Philippe Luchsinger/Präsident mfe weist auf die politische Bedeutung dieses Dachverbandes hin. Seit 2009 vertritt die mfe die Haus- und Kinderärzte Schweiz in politischen und standespolitischen Angelegenheiten. Sie engagiert sich aktiv in Tariffragen wie auch in allen relevanten Themen der Standespolitik und nimmt eine bedeutende Rolle im Gesundheitswesen ein. 
Bisher wurden die Interessen des Berufsverbandes in den Gremien der FMH durch von mfe den Fachgesellschaften zur Verfügung gestellte Sitze und entsprechende Kooperationen wahrgenommen. Gerade nach der Gründung der neuen Fachgesellschaft für Allgemeine Innere Medizin ist es der mfe ein Anliegen, einen offiziellen Platz in der FMH einzunehmen.

\section{Antrag mfe:}

Aufnahme von mfe - Haus- und Kinderärzte Schweiz als mitspracheberechtigte Ärzteorganisation nach Art. 25 Abs. 2 Statuten FMH.

\section{Beschluss:}

Der Antrag wird mit 134 Ja, 2 Nein und 11 Enthaltungen angenommen.

\section{Projekt Coach my Career}

Jürg Unger-Köppel/ZV-Mitglied und Departementsverantwortlicher Stationäre Versorgung und Tarife berichtet über das auf Initiative der FMH, mfe, SIWF, SWIMSA, VLSS und VSAO gemeinsam entwickelte Projekt. Die beruflichen Perspektiven, die sich künftigen Ärztinnen und Ärzten bieten, sind oft weder vorhersehbar noch kontrollierbar. In der Ausbildung kann die Ärzte- schaft das Steuer in die Hand nehmen, vor allem beim Mentoring des ärztlichen Nachwuchses. Dies gilt besonders für die Planung der Weiterbildung und der beruflichen Entwicklung. Die Initianten lancieren deshalb das gemeinsame Projekt mit zwei Angeboten. Ein Angebot ist für die Medizinstudierenden am Ende ihrer Ausbildung. Diese sollen im letzten Jahr mittels Seminaren auf den Berufseinstieg vorbereitet werden (z.B. zeitgemässe Bewerbungsunterlagen erstellen, Strategien bei der Wahl des Faches und der Ausbildungsstätte). Die Veranstaltungen werden von der SWIMSA an den medizinischen Fakultäten organisiert. Ein erstes Seminar findet bereits dieses Jahr an der Universität Zürich statt. Das zweite Angebot der Einzelcoachings richtet sich an Assistenzärzte und -ärztinnen, aber auch junge Oberärzte und -ärztinnen. In Einzelcoachings werden sie von jeweils zwei erfahrenen Kollegen und Kolleginnen bei ihrer Laufbahnplanung beraten, um eine möglichst befriedigende und nachhaltige Lösung für ihr zukünftiges Berufs- und $\mathrm{Fa}$ milienleben $\mathrm{zu}$ finden. Bis heute sind bereits über 30 Anmeldungen für Coaches eingegangen. Die Coachings beginnen ab kommenden September. Der Aufruf für die Mentees erfolgt im Juni. Das Projekt soll

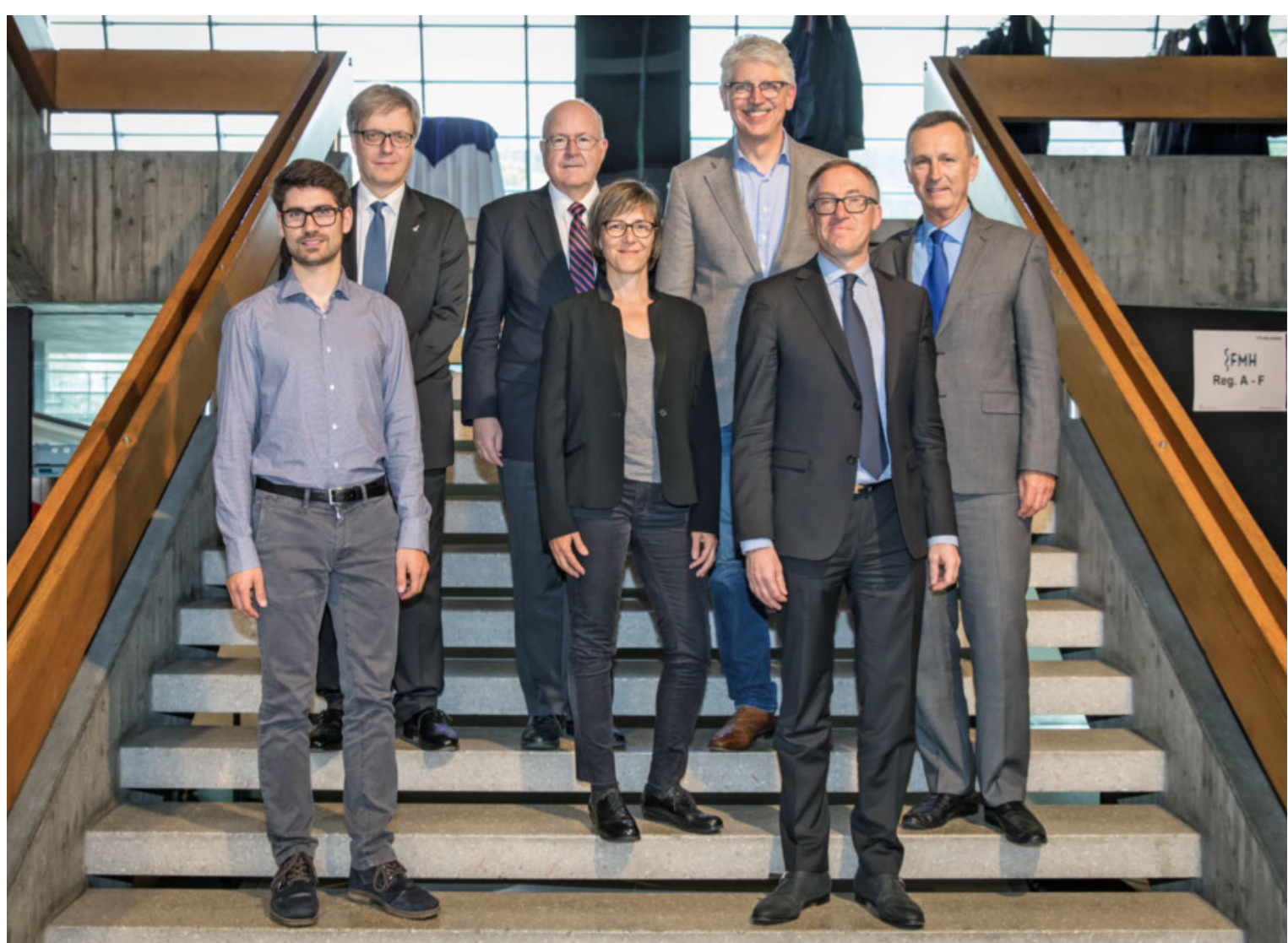

Das Projekt-Team «Coach my Career» bestehend aus Vertretern der FMH, des VSAO, VLSS, mfe und der SWIMSA. Unterstützt wird das Projekt durch das SIWF. 
als Ergänzung zu bereits bestehenden Coachingangeboten, die von den medizinischen Fakultäten, Fachgesellschaften und weiteren Organisationen organisiert werden, verstanden werden.

\section{MARS - Erhebung MAS}

Christoph Bosshard/Vizepräsident FMH und Departementsverantwortlicher Daten, Demographie und Qualität blickt auf die erste, im Juni 2017 abgeschlossene, Datenerhebung im Projekt MAS betreffend Datenjahr 2015 zurück, welche rein statistischen Zwecken galt. Art. 30 KVV, Art. 59a KVG wie auch das Bearbeitungsreglement galten für diese erste Erhebung noch nicht. Die FMH hat zusammen mit dem BFS die Plausibilisierung der Erhebungsdaten durchgeführt und die Auswertung wurde vom BFS am 10.4.2018 publiziert. Eine wichtige Voraussetzung für die Teilnahme der Ärzteschaft an der Erhebung MAS 2015 war, dass die FMH als wichtigste Berufsorganisation sowie ihre Partner (Ärztekasse und NewIndex) die Datenlieferung zu statistischen Zwecken mittels Schnittstellen zu den bestehenden Datensammlungen der Ärztekasse (RoKo), der NewIndex, der FMH und des MedReg unterstützten und so den Unternehmen die Datenerfassung vereinfachten. Ebenfalls wurde so eine hohe Datenqualität gefördert. Erstmals sind somit nun Daten des BFS zu Arztpraxen und ambulanten Zentren verfügbar und die Gesundheitsversorgung einschliesslich ambulanten Bereich kann vertieft dargestellt werden. Die Publikation des BFS vom 10.4.2018 unterstützen die bisher von der FMH gemachten Aussagen in wichtigen Bereichen sehr, was einmal mehr zeigt, dass die Ärzteschaft Transparenz nicht fürchtet, sondern fördert. Gleichzeitig sind der FMH die auch datenschutzrechtlich schutzwürdigen Interessen ihrer Mitglieder sehr wichtig. Die nun verfügbaren vom BFS qualitativ validierten Daten können im Rahmen des Bundesstatistikgesetzes von allen zu statistischen Zwecken beim BFS verlangt werden. Entsprechende Anfragen für Datenlieferungen sind im Zusammenhang mit einem Datenschutzvertrag möglich, wobei lediglich anonymisierte Daten ohne Rückschlussmöglichkeiten auf einzelne Personen und Unternehmen weitergegeben werden.

Die Erhebung der Strukturdaten der Arztpraxen und ambulanten Zentren für das Datenjahr 2017 startet am 11.11.2018. Diese Erfassung dient neu auch den aufsichtsrechtlichen nebst den statistischen Zwecken. Für Leistungserbringer, die an der Erhebung nicht teilnehmen, wird das BFS keine Sanktionen verhängen und auch keine Meldung an kantonale Instanzen erstatten. Die Vorjahresdaten aus der Erhebung 2015 können importiert werden. Wesentlich ist, dass jeder Leis- tungserbringer die Daten zur statistischen oder aufsichtsrechtlichen Weiterbearbeitung freigeben kann. Was die Datenlieferung an das BAG, an die Kantone und Versicherer betrifft, ist gemäss Bearbeitungsreglement folgendes Vorgehen vorgesehen:

Die anonymisierten Daten werden vom BFS den Empfängern auf Gesuch hin übermittelt. Das Gesuch muss den angestrebten Zweck der Datenbearbeitung und die dafür notwendigen Daten genau spezifizieren, in Konformität mit dem vorliegenden Bearbeitungsreglement und gestützt auf Art. 59a KVG und Art. 30 ff KVV. Jeder Empfänger von Daten zu aufsichtsrechtlichen Zwecken hat vor dem Empfang die Verwendungsvereinbarung zu unterzeichnen. Er verpflichtet sich, die erhaltenen Daten nur zum angegebenen Zweck zu verwenden, nicht weiterzugeben und nach Beendigung der Verwendung zu löschen.

Die Datenempfänger sind gemäss Art. $31 \mathrm{KVV}$ verpflichtet, die ihnen weitergegebenen Daten zu löschen, sobald diese zur Erreichung des Zweckes nicht mehr benötigt werden. Sie müssen die Daten gemäss Art. 4 und 5 des Bearbeitungsreglements jedoch spätestens 5 Jahre nach deren Erhalt vernichten.

Christoph Bosshard/ZV-Vizepräsident und Departementsverantwortlicher Daten, Demographie und Qualität bezeichnet die Zusammenarbeit mit dem BFS als konstruktiv und zielgerichtet. Er dankt seiner Abteilungsleiterin, Esther Kraft, wie auch dem Rechtskonsulenten, Michel Meier, für die grosse Unterstützung.

\section{Problemkreis «Ärztinnen und Ärzte in der Doppelrolle»}

Michel Matter/ZV-Mitglied und Departementsverantwortlicher Dienstleistungen und Berufsentwicklung gibt einen Überblick über den an der ÄK vom 4.5.2017 vom Aargauischen Ärzteverband (AAV) eingereichten Antrag. Dabei geht es um die Förderung der Vereinbarkeit von Familie und Beruf sowie die bessere Gestaltung der Aus- und Weiterbildung. Nach einem internen Treffen mit Werner Bauer/Präsident SIWF und Christoph Hänggeli/Geschäftsführer SIWF wurde diese Fragestellung von Jürg Lareida/AAV an der Journée de réflexion vom 20./21.1.2017 in Luzern präsentiert. Die Journée de réflexion ist eine seit Jahren etablierte Einladungsveranstaltung zu Fragen der ärztlichen Bildung. Organisiert wird sie vom SIWF sowie vom Kollegium der Dekane und der Schweizerischen Medizinischen Fakultäten. Der Referent ortet Probleme mit dem heutigen Weiterbildungssystem, welche u.a. ein Hindernis für eine erfolgreiche Vereinbarkeit von Familie und Beruf darstellt. Verbesserungspotenzial ist vor allem hier angezeigt. Das SIWF, die Berufsorganisationen 


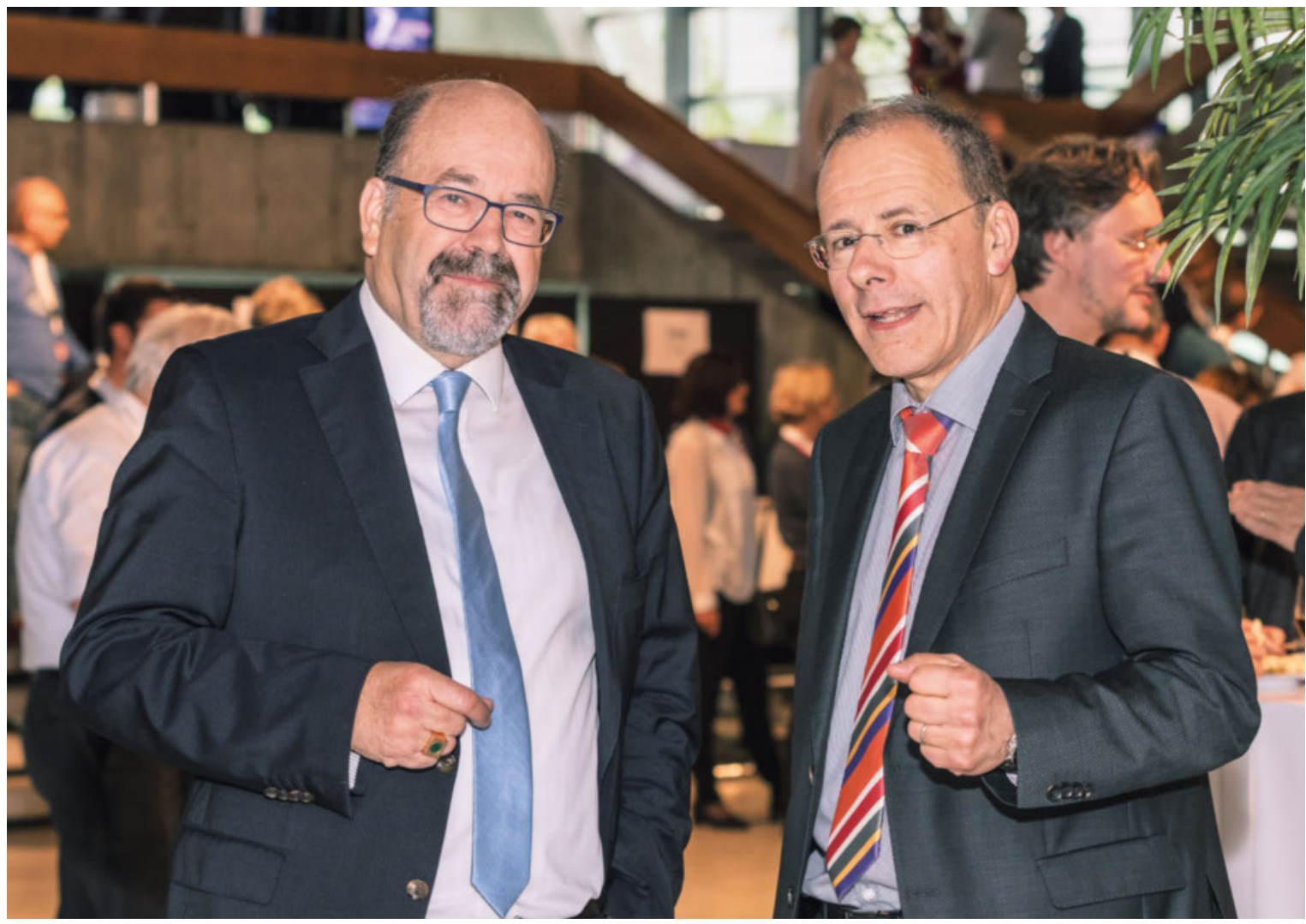

Der Zuger Ständerat und Präsident der SGK-S Joachim Eder im Gespräch mit FMH-Präsident Jürg Schlup.

sowie die medizinischen Fakultäten haben die Anliegen an diesem Anlass aufgenommen und wollen bei der künftigen Entwicklung des Weiterbildungssystems Vorschläge erarbeiten. Das Departement für Dienstleistungen und Berufsentwicklung erachtet diese Lösungen als prioritär, die im Interesse der Ärzte und Ärztinnen, welche zunehmend Teilzeit arbeiten wollen, stehen. Werner Bauer/Präsident SIWF dankt Jürg Lareida/AAV für seine Präsentation an der Journée de réflexion und betont, dass das SIWF die weitere Entwicklung beobachten wird.

\section{Informationen aus ZV, General- sekretariat, SIWF und Abteilungen}

Keine Wortmeldungen.

\section{Varia}

\subsection{Termine ÄK Oktober 2018}

Der Präsident informiert, dass für die Herbst-ÄK die Genehmigung der ambulanten Tarifstruktur traktandiert und die Sitzung somit $1 \frac{1 / 2}{2}$ Tage dauern wird. Er bittet die Delegierten, sich Mittwoch, 24.10.2018, nachmittags, und Donnerstag, 25.10.2018, ganztags, zu reservieren.

\subsection{Termine ÄK Oktober 2019}

Donnerstag, 2.5.2019, und Donnerstag, 31.10.2019

\subsection{Antrag VSAO betreffend Termin ÄK Mai 2020}

Der VSAO regt an, die Frühjahrs-ÄK ab 2020 nicht in der ersten Mai-Woche, sondern in der zweiten Woche zu terminieren.

12.4 Angelo Cannova/AGZ wünscht betreffend Limitationen im neuen TARMED Auskunft. Das Departement Ambulante Versorgung und Tarife wird diesbezüglich mit ihm Kontakt aufnehmen.

Jürg Schlup/Präsident FMH dankt den Mitarbeitenden des Generalsekretariats, unter der Leitung von Beatrice Egger, für die bestens organisierte Tagung sowie allen Delegierten für die zielführende und effiziente Sitzungsarbeit. 


\section{Glossar}

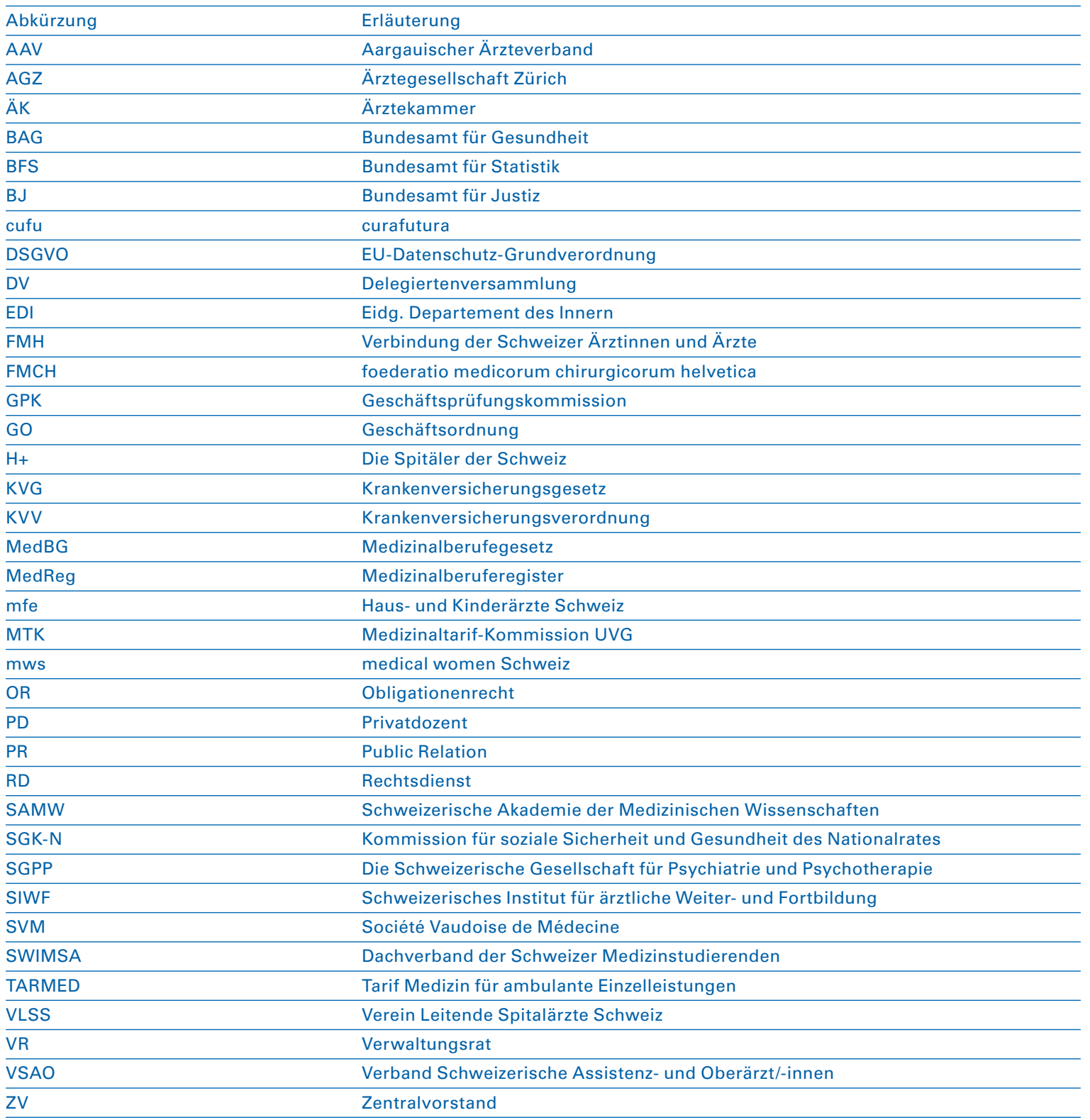

